\title{
On efficient sparse spike coding schemes for learning natural scenes in the primary visual cortex Laurent Perrinet*
}

\author{
Address: Institut de Neurosciences Cognitives de la Méditerranée, CNRS \& Aix-Marseille University, Marseille, France \\ Email: Laurent Perrinet* - Laurent.Perrinet@incm.cnrs-mrs.fr \\ * Corresponding author
}

from Sixteenth Annual Computational Neuroscience Meeting: CNS*2007

Toronto, Canada. 7-12 July 2007

Published: 6 July 2007

BMC Neuroscience 2007, 8(Suppl 2):P206 doi:I0.I I86/I47|-2202-8-S2-P206

(C) 2007 Perrinet; licensee BioMed Central Ltd.

We describe the theoretical formulation of a learning algorithm in a model of the primary visual cortex (V1) and present results of the efficiency of this algorithm by comparing it to the SparseNet algorithm [1]. As the SparseNet algorithm, it is based on a model of signal synthesis as a Linear Generative Model but differs in the efficiency criteria for the representation. This learning algorithm is in fact based on an efficiency criteria based on the Occam razor: for a similar quality, the shortest representation should be privileged. This inverse problem is $\mathrm{NP}$-complete and we propose here a greedy solution which is based on the architecture and nature of neural computations [2]). It proposes that the supra-threshold neural activity progressively removes redundancies in the representation based on a correlation-based inhibition and provides a dynamical implementation close to the concept of neural assemblies from Hebb [3]). We present here results of simulation of this network with small natural images (available at http://incm.cnrs-mrs.fr/Laurent Perrinet/SparseHebbianLearning) and compare it to the Sparsenet solution. Extending it to realistic images and to the NEST simulator http://www.nest-initiative.org/, we show that this learning algorithm based on the properties of neural computations produces adaptive and efficient representations in $\mathrm{V} 1$.

\section{Acknowledgements}

This was work supported by the 6th RFP of the EU (grant no. 15879-FACETS). Simulations use the PyNN software available at http:// pynn.gforge.inria.frl.

\section{References}

I. Olshausen B, Field DJ: Sparse coding with an overcomplete basis set: A strategy employed by VI? Vision Res 1997, 37:33II-3325.

2. Perrinet L: Feature detection using spikes: the greedy approach. J Physiol Paris 2004, 98(4-6):530-539.

3. Hebb DO: The organization of behavior. Wiley, New York; 1949. 